\title{
PLURI: CONSTRUINDO UMA AVALIAÇÃO INTEGRADA PARA O ENSINO MÉDIO INTEGRADO
}

\author{
Paulo Henrique Santana de Oliveira ${ }^{1}$; Laila Lidiane da Costa Galvão²; Fábio Ricardo \\ Mizuno Lemos ${ }^{3}$; Ana Luiza Borges de Paula Nunes ${ }^{4}$; Lucas Fagundes Vaz ${ }^{5}$ \\ ${ }^{1}$ Mestre em Gestão Organizacional pelo Programa de Pós-Graduação em Gestão \\ Organizacional da Universidade Federal de Goiás, Catalão-GO, Brasil. \\ (paulhsdeoliveira@gmail.com) \\ ${ }^{2}$ Mestra em Gestão Organizacional pelo Programa de Pós-Graduação em Gestão \\ Organizacional da Universidade Federal de Goiás, Catalão-GO, Brasil. \\ ${ }^{3}$ Doutor em Educação pela Universidade Federal de São Carlos, \\ São Carlos-SP, Brasil. \\ ${ }^{4}$ Mestra em Ecologia e Conservação de Recursos Naturais, pela UFU, \\ Uberlandia/MG. \\ ${ }^{5}$ Especialista em Gestão de Tecnologia da Informação pela Universidade Cândido \\ Mendes, Rio de Janeiro-RJ, Brasil.
}

\section{Recebido em: 06/04/2018 - Aprovado em: 10/06/2018 - Publicado em: 20/06/2018 DOI: 10.18677/EnciBio_2018A134}

\begin{abstract}
RESUMO
Esse trabalho teve o objetivo de relatar a experiência de uma ação acadêmica que envolveu práticas e conhecimentos de forma interdisciplinar. Esta ação denomina-se Pluri e constitui-se em uma atividade avaliativa utilizada pelos Cursos Técnicos Integrados ao Ensino Médio do Instituto Federal do Triângulo Mineiro - Campus Patrocínio, para propiciar a integração entre a formação propedêutica e a formação profissional e possibilitar um desenvolvimento interdisciplinar e contextualizado aos discentes, a partir do diálogo entre os conteúdos de diversas unidades curriculares, rompendo gradativamente com o ensino fragmentado. O Pluri é aplicado trimestralmente, desde o ano de 2014, a todos os estudantes dos Cursos Técnicos Integrados ao Ensino Médio (Administração; Eletrônica e Manutenção e Suporte em Informática) e faz parte do rol das avaliações somativas dos cursos. A avaliação é composta por questões elaboradas pelos docentes de duas ou mais disciplinas dos cursos, além de questões sobre atualidades e de uma produção textual. Neste trabalho foi apresentada uma análise comparativa sob a ótica qualitativa dos resultados do Pluri 2014 a 2017, tomando por base: documentos institucionais, depoimentos de estudantes e parecer da equipe pedagógica. Como principal resultado foi constatado que a busca de pontos de integração entre as disciplinas para a construção das questões do Pluri abriu portas para a interdisciplinaridade também em outras atividades educacionais.
\end{abstract}

PALAVRAS-CHAVE: avaliação integrada, cursos técnicos integrados ao ensino médio, interdisciplinaridade. 


\title{
PLURI: MAKING AN INTEGRATED EVALUATION FOR INTEGRATED HIGH SCHOOL
}

\begin{abstract}
This work had the objective of reporting the experience of an academic action that involved practices and knowledge in an interdisciplinary way. This action is called Pluri and is an evaluation activity used by the Technical Courses Integrated to High School of the Instituto Federal do Triângulo Mineiro - Campus Patrocínio, to propitiate the integration between the preparatory training and the professional formation and to enable an interdisciplinary and contextualized to the students, starting from the dialogue between the contents of several curricular units, breaking gradually with the fragmented teaching. Pluri is applied quarterly, since 2014, to all students of the Technical Courses Integrated to High School (Administration, Maintenance and Support in Informatics, Electronics) and is part of the list of summative evaluations of the courses. The evaluation is composed by questions elaborated by the teachers of two or more disciplines of the courses, besides questions about current and textual production. In this work a comparative analysis was presented under the qualitative perspective of the results of the Pluri 2014 to 2017, taking as a base: institutional documents, student testimonials and opinion of the pedagogical team. As a main result it was verified that the search for points of integration between the disciplines for the construction of the Pluri issues opens doors to the interdisciplinarity also in other educational activities.
\end{abstract}

KEYWORDS: integrated evaluation, technical courses integrated to high school, interdisciplinarity.

\section{INTRODUÇÃO}

A avaliação da aprendizagem, segundo Fazenda et al. (2010) consiste em um processo inerente ao aprender relacionado diretamente aos objetivos que são estabelecidos como parâmetros a serem alcançados em relação às atividades curriculares trabalhadas. Ao avaliar um estudante é fundamental considerar diversos elementos constantes no processo de aprendizagem. Desse modo, reconhecer as diversas formas de ensino e de aprendizagem é essencial para que se compreendam os conhecimentos construídos no decorrer do período estudado.

É preciso ir além da quantificação dos conteúdos apreendidos e considerar as diferenças individuais existentes em sala de aula assim como o conhecimento prévio dos estudantes. Na visão de Darsie (1996) tais fatores contribuem para uma visão mais ampliada das aprendizagens concretizadas e para a construção de um processo de avaliação mais eficaz. Segundo Alcântara et al. (2016):

O aluno é elemento ativo nos processos de ensino e de aprendizagem, como é também o professor. Portanto, a relação entre ambos deve ser de constante interação, para que ocorra a produção do conhecimento. A discussão sobre os instrumentos utilizados pode ser um caminho para a conscientização, ajudando tanto os professores, quanto os alunos a melhor compreender o processo de avaliação.

Segundo Darsie (1996) o contexto atual impõe a necessidade da reflexão sobre as ações avaliativas desenvolvidas no cotidiano escolar. É constante a 
necessidade de refletir sobre as práticas pedagógicas realizadas na escola, uma vez que, as mudanças nas concepções de avaliação só se tornam possíveis mediante remodelações no processo de ensinar e aprender. Diante dessas argumentações, percebe-se a necessidade de uma inovação na ação pedagógica, em todas as suas categorias, voltada para a valorização do diálogo e a integração entre os componentes curriculares visando identificar as possibilidades de aproximação e complementação.

O presente trabalho teve por objetivo apresentar um relato de experiência sobre a criação e implantação de uma avaliação integrada voltada ao Ensino Médio Integrado ao Técnico, bem como, as contribuições desta iniciativa.

\section{ENSINO MÉDIO INTEGRADO}

O Ensino Médio Integrado, segundo Frigotto et al. (2012) surgiu no Brasil a partir de 2004, particularmente, a partir do novo contexto do Decreto № 5.154 de 23/07/2004, que consentiu o curso de matrícula única que antes inexistia. O Ensino Médio Integrado passou a ser um curso de nível médio mais completo e mais bem articulado, pois abrange a formação básica para a cidadania relacionada com a formação profissional de nível técnico em um único curso, o que garante ao aluno, a formação cidadã, a possibilidade de ingresso no mercado de trabalho e a possibilidade de continuidade dos estudos.

Araújo e Silva (2017) explanam que a proposta de Ensino Médio Integrado à educação profissional contrapõe-se ao modelo de ensino hegemônico no Brasil, que se caracteriza por um currículo fechado e pouco flexível, que busca preparar os jovens apenas para exames e ingresso no superior, com um viés centrado no conteúdo e nas disciplinas, que inviabiliza a construção de projeto pedagógicos inovadores.

Segundo Brasil (2009), é preciso estimular novas formas de organização das disciplinas, o que também envolve a inovação na avaliação da aprendizagem, articulando-as com atividades integradoras, a partir das inter-relações existentes entre os eixos constituintes do Ensino Médio, o trabalho, a ciência, a tecnologia e a cultura. Conforme Luckesi (2013) para que se utilize corretamente a avaliação no processo ensino-aprendizagem no contexto escolar, importa estabelecer um parâmetro mínimo de conhecimentos, habilidades e hábitos que o educando deverá adquirir.

No entanto, é preciso ir além da quantificação dos conteúdos ensinados/aprendidos e considerar as diferenças individuais presentes na sala de aula e o conhecimento prévio dos alunos como fatores que contribuem para uma visão mais ampliada das aprendizagens. A valorização do diálogo e a integração entre os componentes curriculares, visando identificar as possibilidades de aproximação e complementação, colaboram para 0 estabelecimento da interdisciplinaridade, tema relevante e crescente nos estudos relacionados ao ensino médio (MOZENA; OSTERMANN, 2014).

França (2014) destaca que a interdisciplinaridade é uma categoria de ação do fazer reflexivo, do acontecer entre duas ou mais pessoas e objetos, por meio da qual é possível a integração e a unicidade do conhecimento a partir de zonas de permeabilidade de diálogos e leitura de diferentes mundos. O objetivo deste trabalho foi relatar a experiência de uma ação acadêmica que envolveu práticas e conhecimentos de forma interdisciplinar. 


\section{MATERIAL E MÉTODOS}

Este estudo consistiu em um relato de experiência vivenciado por autores do presente trabalho durante o desenvolvimento, a implantação e consolidação da Atividade Educacional Pluri como ferramenta pedagógica de avaliação integrada na instituição Instituto Federal de Educação Ciência e Tecnologia do Triângulo Mineiro Campus Patrocínio no período de 2014 a 2017.

O desenvolvimento desta inovação educacional se deu antes do início das primeiras turmas dos Cursos Técnicos Integrados ao Ensino Médio, mais especificamente durante a criação dos Projetos Pedagógicos dos Cursos, no Instituto Federal do Triângulo Mineiro - Campus Patrocínio tendo início no ano de 2014. Tal período foi favorável para a implantação de uma nova estratégia educacional interdisciplinar que conseguisse verificar competências e habilidades dos estudantes a cada período letivo. A implantação desta inovação no início dos novos cursos teve o intuito de dirimir possíveis resistências que pudessem surgir por parte da equipe educacional que atuaria nas salas de aula.

Durante sua implantação, o Pluri foi adotado como método de avaliação em todos os Cursos Técnicos Integrados ao Ensino Médio da instituição sendo: Administração; Eletrônica e Manutenção e Suporte em Informática. Foi constituída uma comissão para organização, coleta das questões, diagramação, aplicação e correção da atividade avaliativa e os autores deste trabalho participaram ativamente da comissão desde a primeira edição.

A comissão supracitada manteve atas de registros dos trabalhos e decisões tomadas e foi criado um e-mail para divulgação de boletins informativos sobre o PLURI entre docentes e alunos. Estes documentos foram utilizados como fonte de pesquisa para construção deste estudo, assim como livros e artigos com temáticas relacionadas às avaliações da aprendizagem, avaliação interdisciplinar e ensino médio integrado compondo uma pesquisa bibliográfica e documental que fundamentou a criação do presente relato de experiência.

\section{RESULTADOS E DISCUSSÃO}

A experiência com o Pluri ocorreu entre os anos letivos de 2014 a 2017, e o o relato toma por base: documentos institucionais, depoimentos de estudantes e parecer da equipe pedagógica. O Pluri é uma avaliação integrada e interdisciplinar, centrada na verificação de competências e habilidades, composta por 40 questões de múltipla escolha, e uma redação cujo formato assemelha-se àquele praticado no ENEM, que, conforme salientam Mozena e Ostermann (2014), inseriu-se em uma proposta interdisciplinar a partir das mudanças ocorridas em 2009.

A avaliação procura ainda manter os estudantes atualizados e informados quantos aos principais acontecimentos correntes e verificar a aquisição instrumental de conhecimentos técnicos relacionados à habilitação profissional, visando colaborar no atendimento das necessidades dos estudantes quanto à preparação para a vida, para 0 trabalho e para a continuidade dos estudos, nesta ordem de prioridade. $O$ Pluri se trata de uma inovação educacional criada pela unidade de ensino supracitada, não havendo, portanto trabalhos acadêmicos anteriores a este, sobre este tema.

O Pluri tem como objetivos principais: oportunizar aos estudantes do Ensino Médio Integrado, o contato com uma avaliação centrada na verificação de competências e habilidades, condizente com a sistemática adotada no ENEM. Incrementar uma atividade de forte cunho integrador e interdisciplinar necessária para a adequada preparação para os processos seletivos de cursos superiores, para 
o mercado de trabalho e para a Vida. E, por fim, reduzir o número de avaliações trimestrais desafogando a agenda de atividades dos estudantes, contribuindo para reduzir a repetência e a evasão. Tais objetivos têm consonância com o pensamento de Freitas et al. (2017), quando apontam que a avaliação não deve incorporar apenas objetivos escolares, das matérias ensinadas, mas também os ligados à função social da escola no mundo atual.

O Pluri possui a valoração de sete pontos, o que representa cerca de $20 \%$ da nota total do trimestre letivo, sendo esta pontuação distribuída da seguinte forma: quatro pontos para o caderno de questões e três pontos atribuídos para a avaliação de uma redação. Após a correção, o total da pontuação atribuída a cada estudante é replicada para todas as unidades curriculares dos cursos.

A organização do Pluri fica ao encargo de uma comissão de servidores e discentes designada pelo diretor do Campus para cada uma das funções a seguir: Análise das questões; Revisão; Formatação; Impressão das Avaliações; Definição de Aplicadores e Corretor; Listas de Presença; Organização das salas; Recursos; Divulgação do Gabarito; Correção; Divulgação Final das Notas. É importante ressaltar que os estudantes membros da comissão, não tem acesso à avaliação antes da aplicação. A participação é de extrema importância a fim de colaborar com o processo decisório geral sobre a operacionalização da avaliação envolvendo decisões, tais como: datas e horários de aplicação, avaliação de pareceres sobre recursos interpostos por estudantes com relação às questões, dentre outras.

Para a elaboração de questões, os professores são divididos em grupos de acordo com as respectivas áreas de atuação onde cada grupo tem um representante que é responsável por repassar à comissão as questões elaboradas pelo grupo. Cada professor deve elaborar duas ou três questões para o Pluri, sendo que há uma seleção entre as questões que serão utilizadas para composição da avaliação. Os professores das disciplinas que têm uma carga horária menor elaboram duas questões e os professores das disciplinas que tem carga horária maior ficam responsáveis pela elaboração de três questões.

As questões adicionais são armazenadas em um Banco de Questões, e podem vir a ser utilizadas em próximas edições da avaliação integrada. As provas são compostas de 40 questões e uma redação. É importante ressaltar que as questões são elaboradas de forma interdisciplinar entre dois ou mais professores com o intuito de estimular a busca por pontos de integração entre os conteúdos de suas disciplinas, proporcionando oportunidades para que outras atividades interdisciplinares sejam realizadas a partir da descoberta de convergências.

De acordo com a equipe pedagógica que faz a análise da avaliação todas as questões têm um nível de interdisciplinaridade, contudo algumas têm nível superficial (através de artifícios como a contextualização) e outras, um nível mais aprofundado, demonstrando que os professores estão se habituando a buscar esta estratégia educacional. São realizados treinamentos e reuniões periódicas a fim de conscientizar a equipe da importância da busca por estratégias que promovam a inter e transdisciplinaridade na educação e orientá-la sobre os melhores caminhos a seguir.

Com relação à aplicação da avaliação, na data da realização os discentes são organizados em ordem alfabética, em uma lista geral com todos os estudantes dos Cursos Integrados e as salas são organizadas de acordo com essa lista, mesclando alunos de todos os cursos e séries. As carteiras são identificadas com os nomes dos estudantes e estes podem adentrar nas salas em um horário pré-definido, com tolerância de no máximo 30 minutos. Cada sala fica sob a responsabilidade de dois 
aplicadores, que devem transmitir as instruções para a avaliação e supervisionar os estudantes durante a realização da atividade.

As avaliações são entregues lacradas aos aplicadores, que juntamente com os primeiros alunos de três filas assinam um termo comprovando o recebimento das avaliações lacradas. Logo após, as instruções para a realização são transmitidas aos alunos e cada um recebe o caderno com 40 questões (valendo 0,1 pontos) com quatro alternativas cada, sendo apenas uma correta.

Os alunos começam a responder o caderno de questões ao mesmo tempo, podendo deixar o local de prova somente depois de decorridas duas horas e meia do início da aplicação e não podem levar o caderno de questões ao deixarem definitivamente a sala. Os três alunos que restarem na sala assinam um termo confirmando que estavam presentes quando o último aluno entregou o caderno de questões e que não foi favorecido por nenhum dos aplicadores. Ao término da avaliação os alunos estão liberados das demais atividades escolares.

No que se refere à correção, uma subcomissão é responsável por verificar os gabaritos com a máscara de correção. Após a revisão, o caderno de provas é devolvido aos discentes. As redações são digitalizadas e enviadas para os dois corretores da área de linguagens, que separadamente fazem a correção. É feita uma média das notas entres esses corretores, e assim se dá a nota oficial da redação. As redações são entregues em dias diferentes dos cadernos de questões.

A divulgação do gabarito preliminar ocorre em até uma semana após a entrega do caderno de questões aos alunos. Os alunos podem interpor recursos em até dois dias após a divulgação do gabarito preliminar. O recurso é analisado pela subcomissão. Se esse recurso for deferido, ele é divulgado e o gabarito corrigido.

Durante o período mencionado foram aplicadas 12 edições do PLURI. Houve treinamentos aos professores para elaboração de questões de forma interdisciplinar. Segundo parecer da equipe pedagógica há a necessidade de estabelecer uma agenda de treinamentos com relação a esta temática. Como há um alto nível de rotatividade da equipe há uma discrepância entre o nível de preparação dos novos professores em relação aos professores que já participaram de várias edições do Pluri, gerando um déficit de qualidade em algumas questões.

$\mathrm{Na}$ área propedêutica a elaboração foi dividida entre as grandes áreas tal como acontece no ENEM sendo elas: Ciências da Natureza e suas Tecnologias, Ciências Humanas e suas Tecnologias, Linguagens, Códigos e suas Tecnologias e Matemática. Hartmann e Zimmermann (2007) corroboram que a interdisciplinaridade representa uma nova etapa para o desenvolvimento do conhecimento, estabelecendo que as disciplinas se unam por meio de uma constante articulação. Tal divisão incentivou maior integração entre os professores de cada área a fim de realizarem outras atividades de forma interdisciplinar. Como fruto desta interação surgiram projetos, tais como: a Gincamática, o Cine History, o Sarau Literário, o I Encontro de Linguagens dentre outros.

$\mathrm{Na}$ área técnica os professores foram orientados a elaborar questões interdisciplinares com os professores das disciplinas propedêuticas, tal interação proporcionou à equipe a percepção da importância da integração entre as diferentes áreas da vida para solucionar questões do cotidiano profissional. Segundo Luckesi (2013) é necessário que os professores estejam dispostos a aprender uma nova metodologia para avaliar a aprendizagem, pois assim como os discentes se não se dispuser a aprender, não haverá aprendizagem.

Foi designada uma comissão para elaboração das questões de atualidades e temas das redações. Como resultado do trabalho desta equipe o PLURI $1^{\circ}$ Trimestre 
de 2016 teve como tema de redação: Caminhos para se combater a Intolerância Religiosa no Brasil. Este foi o tema da redação do ENEM do mesmo ano proporcionando aos estudantes um bom desempenho, por já terem feito uma redação com o tema.

Em 2014 foi criado um concurso entre os estudantes para definição do logotipo e do nome da atividade avaliativa. No ano de 2015 o Campus recebeu no Ensino Médio Integrado uma aluna cega gerando a necessidade da transcrição da avaliação PLURI em Braille o que foi realizado através de uma parceria com o Centro de Apoio à Pessoa com Deficiência Visual da cidade de Uberaba/MG. As avaliações são enviadas por e-mail para os transcritores na cidade e o motorista do Campus busca as provas já impressas em Braille para aplicação à aluna que fez a avaliação sem a necessidade de um aplicador leitor. Por dois semestres diferentes, dentro do período do curso técnico integrado no qual está matriculada, a estudante apresentou a melhor pontuação nas questões fechadas dentre todos os estudantes.

Ao findar o ano letivo de 2016, as primeiras turmas de formandos que viveram a trajetória do desenvolvimento do PLURI puderam avaliar o impacto da realização desta atividade através de sua preparação para realização do ENEM. Foram coletados diversos depoimentos que confirmaram as impressões positivas.

\section{CONCLUSÃO}

O presente estudo atingiu seu objetivo que foi o de relatar a experiência vivenciada por docentes, técnico-administrativos e alunos tendo iniciado as atividades do PLURI no ano de 2014. Verificou-se que a Avaliação Integrada PLURI foi uma experiência positiva e contribuiu para a construção de um Ensino Médio realmente Integrado, através do oferecimento de uma perspectiva de interdisciplinaridade. O desenvolvimento de uma avaliação interdisciplinar propiciou maior integração entre os professores das disciplinas impulsionando a busca de convergências entre os conteúdos para realização de outras atividades para além da avaliação.

Observou-se que a avaliação realmente colaborou com a redução do número de avaliações trimestrais, desafogando a agenda de atividades dos estudantes, se tornando um fator relevante para permanência e êxito dos alunos. Como os cursos são integrais, a agenda dos estudantes frequentemente é cheia de atividades. Os cursos possuem entre 15 e 18 disciplinas, o que gera um acúmulo de provas e trabalhos e a necessidade da dedicação de muitas horas de estudo fora da escola. A avaliação PLURI substitui a terceira avaliação de cada disciplina no trimestre, reduzindo então até 54 avaliações por ano (nas turmas que possuem 18 disciplinas/ano).

Conclui-se que o modelo de avaliação desenvolvido é um bom instrumento para ser implantado em outras instituições educacionais, desde que respeitadas as individualidades de cada ambiente.

\section{REFERÊNCIAS}

ALCÂNTARA, L. A. G.; QUARTIERI, M. T.; CARREIRA, S. P. G.; AMADO, N. M. P.; DULLIUS, M. M. Avaliação da aprendizagem escolar na perspectiva dos alunos. Interfaces da Educação, v. 7, n. 20, p. 07-21, 2016. Disponível em: $<$ http://periodicosonline.uems.br/index.php/interfaces/article/download/1153/1079>.

ARAÚJO, A. C.; SILVA, C. N. N. Ensino Médio Integrado no Brasil: fundamentos, práticas e desafios. Brasília: IFB, 2017. 
BRASIL. Ministério da Educação. Parecer CNE/CP no 11/2009: Proposta de experiência curricular inovadora do Ensino Médio. Disponível em: $<$ http://portal.mec.gov.br/dmdocuments/parecer_minuta_cne.pdf>. Acesso em: 30 abr. 2018.

DARSIE, M. M. P. Avaliação e aprendizagem. Cadernos de Pesquisa, n. 99, p. 4759, nov. $1996 . \quad$ Disponível em: <http://publicacoes.fcc.org.br/ojs/index.php/cp/article/view/785/797>.

FAZENDA, I. C. A.; KIECKHOEFEL, L.; PEREIRA, L. P.; SOARES, A. Z. Avaliação e interdisciplinaridade. Revista Interdisciplinaridade, v. 1, n. 0, p. 23-37, out. 2010. Disponível em: $<$ https://revistas.pucsp.br/index.php/interdisciplinaridade/article/view/16142/12179>.

FRANÇA, O. A. V. Ação. In: FAZENDA, I.C.A. (Org.). Interdisciplinaridade: pensar, pesquisar e intervir. São Paulo: Cortez, 2014. p. 27-34.

FREITAS, L. C.; SORDI, M. R. L.; MALAVASI, M. M. S.; FREITAS, H. C. L. Avaliação educacional: caminhando pela contramão. Petrópolis: Vozes, 2017.

FRIGOTTO, G.; CIAVATTA, M.; RAMOS, M. (Orgs.). Ensino médio integrado: concepções e contradições. 3. ed. São Paulo: Cortez, 2012.

HARTMANN, A. M.; ZIMMERMANN, E. O trabalho interdisciplinar no Ensino Médio: a reaproximação das "Duas Culturas". Revista Brasileira de Pesquisa em Educação em Ciências, v. 7, n. 2, p. 1-16, 2007. Disponível em: $<$ https://seer.ufmg.br/index.php/rbpec/article/view/2237/1636>.

LUCKESI, C.C. Avaliação da aprendizagem escolar: estudo e proposições. São Paulo: Cortez, 2013.

MOZENA, E. R.; OSTERMANN, F. Uma revisão bibliográfica sobre a interdisciplinaridade no ensino das ciências da natureza. Revista Ensaio, v. 16, n. 02, p. 185-206, maio-ago. 2014. Disponível em: <http://dx.doi.org/10.1590/198321172014160210>. Doi: 10.1590/1983-21172014160210. 